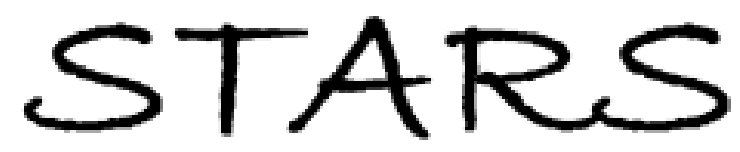

University of Central Florida

STARS

$1-1-2008$

\title{
Numerical analysis of acoustic wave propagation in layered carbon nanofiber reinforced polymer composites
}

Li Sun

Yong Yu

Gangbing Song

Jihua Gou

University of Central Florida

Find similar works at: https://stars.library.ucf.edu/facultybib2000 University of Central Florida Libraries http://library.ucf.edu

This Article is brought to you for free and open access by the Faculty Bibliography at STARS. It has been accepted for inclusion in Faculty Bibliography 2000 s by an authorized administrator of STARS. For more information, please contactSTARS@ucf.edu.

\section{Recommended Citation}

Sun, Li; Yu, Yong; Song, Gangbing; and Gou, Jihua, "Numerical analysis of acoustic wave propagation in layered carbon nanofiber reinforced polymer composites" (2008). Faculty Bibliography 2000s. 1030. https://stars.library.ucf.edu/facultybib2000/1030

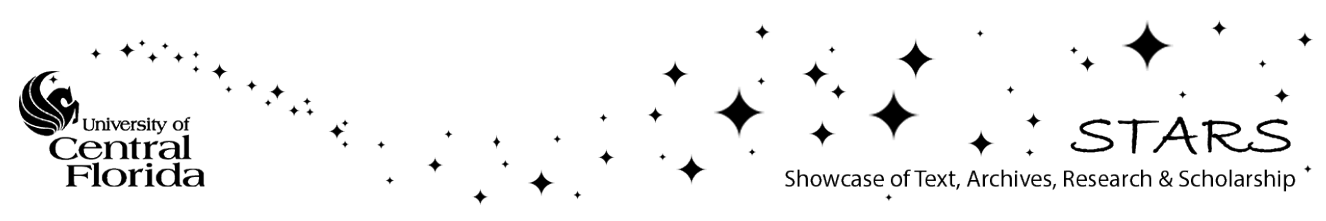




\section{Numerical analysis of acoustic wave propagation in layered carbon nanofiber reinforced polymer composites}

Cite as: J. Appl. Phys. 104, 043522 (2008); https://doi.org/10.1063/1.2973039

Submitted: 07 April 2008 . Accepted: 23 June 2008. Published Online: 27 August 2008

Li Sun, Yong Yu, Gangbing Song, and Jihua Gou

\section{ARTICLES YOU MAY BE INTERESTED IN}

Shock-wave propagation through pristine $a$-SiC and carbon-nanotube-reinforced $a$-SiC matrix composites

Journal of Applied Physics 106, 014311 (2009); https://doi.org/10.1063/1.3152587

Free transverse vibration of the fluid-conveying single-walled carbon nanotube using nonlocal elastic theory

Journal of Applied Physics 103, 024302 (2008); https://doi.org/10.1063/1.2822099

Wave propagation in carbon nanotubes via nonlocal continuum mechanics

Journal of Applied Physics 98, 124301 (2005); https://doi.org/10.1063/1.2141648

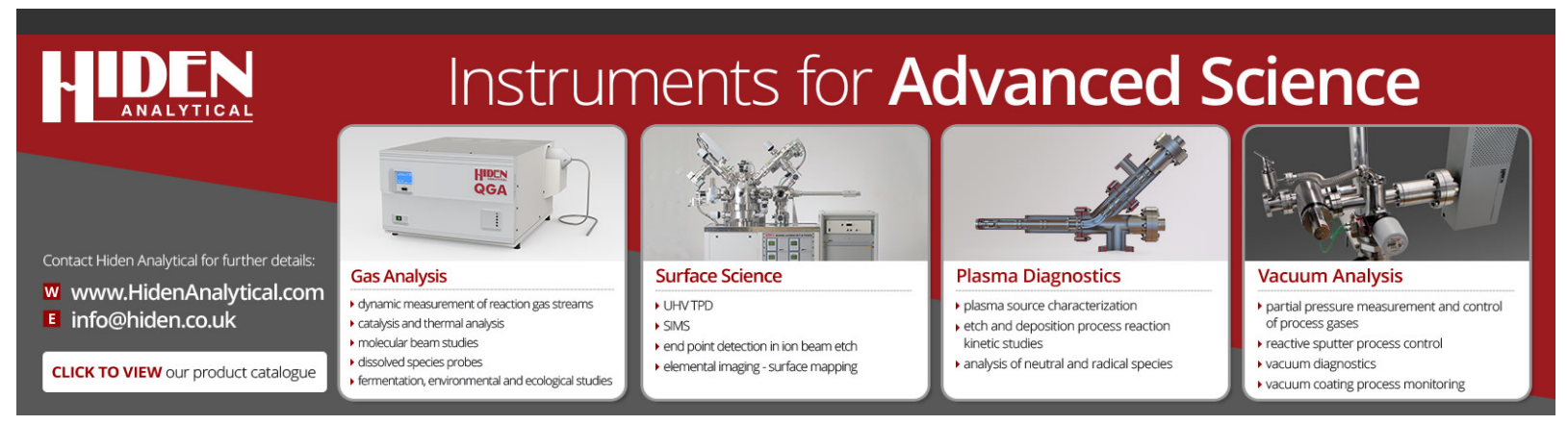




\title{
Numerical analysis of acoustic wave propagation in layered carbon nanofiber reinforced polymer composites
}

\author{
Li Sun, ${ }^{1, a)}$ Yong Yu, ${ }^{1}$ Gangbing Song, ${ }^{1}$ and Jihua $\mathrm{Gou}^{2}$ \\ ${ }_{1}^{1}$ Department of Mechanical Engineering, University of Houston, Houston, Texas 77204, USA \\ ${ }^{2}$ Department of Mechanical, Materials and Aerospace Engineering, University of Central Florida, Orlando, \\ Florida 32816, USA
}

(Received 7 April 2008; accepted 23 June 2008; published online 27 August 2008)

\begin{abstract}
Polymer composites reinforced by carbon nanofibers (CNFs) in the form of paper sheet show significant vibration and acoustic damping improvement when compared to pure matrix materials. Without looking into the microscopic energy dissipation mechanisms, this paper analyzes the wave propagation in the composites from a macroscopic point of view. The CNF nanocomposites in this study were treated as stacking of alternating layers of pure polymer and CNF reinforced polymer. Analyses of acoustic wave propagation focused on revealing the effects of acoustic impedance discontinuity at the interfaces of the layered structure. Plane wave transmission coefficient has been calculated as a function of the number of the layer repeats and thickness at different wave frequencies. Oscillations in the transmission coefficient have been observed when the acoustic wavelength is on the same order of the bilayer thickness, indicating the possibility of designing the nanocomposite structure to optimize noise reduction characteristics. The numerical analysis converges with effective media theory when acoustic wavelength is much larger than the layer thickness. (C) 2008 American Institute of Physics. [DOI: 10.1063/1.2973039]
\end{abstract}

\section{INTRODUCTION}

Fiber reinforced polymer matrix composites (PMCs) are widely used due to their light weight, high specific strength, corrosion/fatigue resistance, and chemical stability. Starting from the 1950s, high performance PMCs reinforced by continuous carbon microfibers or carbon black particles have been developed for aerospace and military applications. These composites now can be found in commercial aircrafts, industrial structures such as wind blades, pressure vessels, and sports and leisure equipments. ${ }^{1}$ With the discovery of carbon nanomaterials in 1980s, there have been increasing interests and efforts in the development and application of PMCs using carbon nanostructures. Majority of the research have been focused on using carbon nanotubes (CNTs) as fillers to take advantage of their large surface area, superior mechanical properties, unique electrical and thermal properties, and thermal/chemical stability. Recent studies showed that these CNT-PMCs could also have improved vibration and acoustic damping properties. ${ }^{2-6}$ Vibrations are normally undesirable for structures, due to the need for structural stability and dynamic response, position control, and durability. At the same time, long-term exposure to even relatively low levels of acoustic signals has been shown to be potentially harmful to humans. Thus both vibration and acoustic damping are important issues for PMC applications.

Energy dissipation in composites can come from the intrinsic viscoelasticity of the matrix and filler, as well as the dynamic interface interactions between the filler and the matrix. Equally important, the wave propagation and reflection can also influence performance of the material. In most of the recent studies, PMCs with nanoscale fillers have been

${ }^{a)}$ Electronic mail: 1sun4@uh.edu. synthesized by direct mixing $^{7-10}$ or in situ polymerization ${ }^{11,12}$ in order to control nanomaterial distribution [Fig. 1(b)]. Theoretical analyses of the wave propagation and attenuation showed that in composites with uniformly distributed fillers, when the acoustic wavelength was several orders larger than the filler characteristic size, Rayleigh-like scattering dominates and the acoustic attenuation caused by nanoscale fillers is negligible. ${ }^{13,14}$ Wave attenuation due to scattering only becomes significant when the size of the inclusions is comparable with acoustic wavelength. ${ }^{15-18}$

In this study, loose carbon nanofibers (CNFs) were first assembled into interconnect and self-supportive sheets [Fig. $1(\mathrm{c})]$ before being incorporated into PMCs. ${ }^{19-21}$ This approach not only improves the efficiency of material handling and synthesis reproducibility, more importantly, the porosity and the electrical conductivity CNF sheets can be controlled. After introducing carbon paper sheets, the PMCs showed significant vibration damping ratio increase. ${ }^{22}$ The introduction of CNFs sheet as reinforcement creates drastic differences in the mechanical responses of pure polymer and rein-

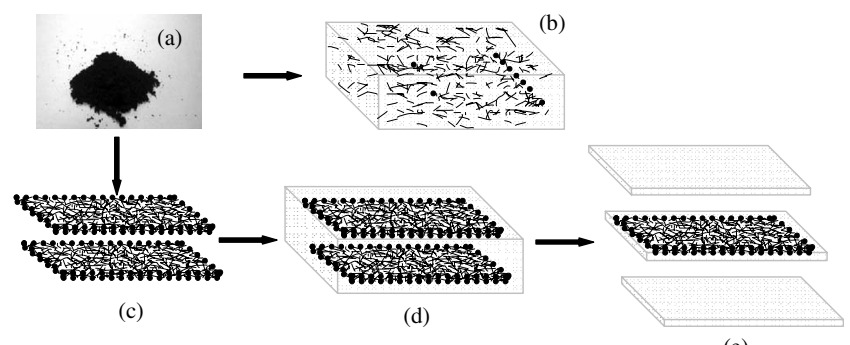

(e)

FIG. 1. Schematics of the two CNF structures. (a) Loose powerlike CNFs, (b) uniformly distributed composite, (c) assembled CNF sheets, (d) composites reinforced by $\mathrm{CNF}$ sheets, and (e) CNF sheet reinforced composite modeled as layered structure. 


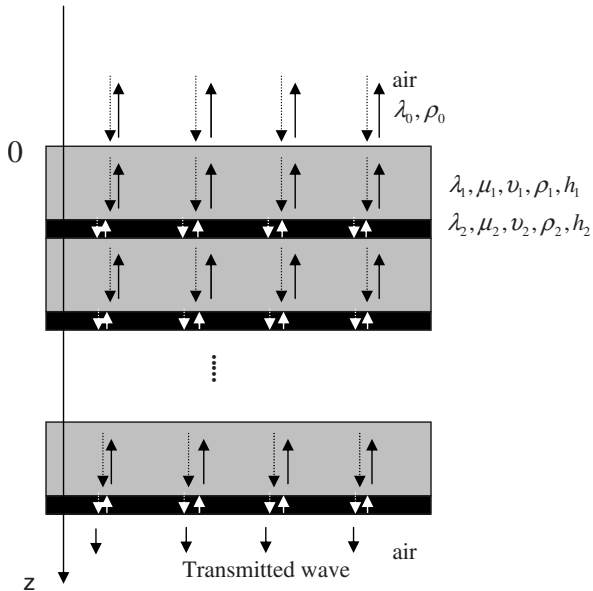

FIG. 2. Schematics of the normal incident plane wave propagating through a layered structure.

forced layers and thus results in discontinuity in acoustic impedance at the interfaces. Considering the similar wave propagation characteristics as photonic structures, ${ }^{23,24}$ this paper reports on the study of wave propagation in a layered structure [Fig. 1(e)] to evaluate the effects of these CNF sheets in the matrix.

\section{ACOUSTIC WAVE PROPAGATION THEORY}

The wave scattering cross section of one single spherical obstacle is proportional to $k^{4} a^{6}$, where $k(=2 \pi / \lambda, \lambda$ is the wavelength) is the wave vector and $a$ is the particle radius. When the particle size is much smaller than the wavelength, the normalized scattering cross section in the Rayleigh limit is very small and independent of particle shape. Due to the fact that acoustic wavelength is normally (even for supersonic frequencies) several orders of magnitude larger than the CNF sizes, the continuous solid media approximation is valid where the composite structure can be viewed as the stacking of two types of homogenous layers. Multiple scattering becomes important only when the scatter size becomes comparable to the wavelength. Discussion on the acoustic wave propagation in a medium containing a random array of obstacles (or scatters) can be found in Ref. 17.

To simplify the discussion, only the normal incident plane wave propagation is considered here. In this case, only longitudinal wave propagates in the material when continuous medium approximation can be satisfied. The normal incident plane acoustic wave propagation in a bilayer structure with infinite lateral dimensions is considered (Fig. 2). A fixed bilayer structure is assumed to exist throughout the sample with perfect interfacial bonding. Since all plane acoustic waves can be represented by the superposition of a series of plane harmonic waves through Fourier transformation, the problem is simplified by investigating plane harmonic wave propagation.

Based on the wave propagation theory, ${ }^{25,26}$ the acoustic wave field in the air can be described by a displacement function $u(z, t)$ when the wave is propagating along the $z$ direction as

$$
u(z, t)=\frac{\partial \phi(z, t)}{\partial z}
$$

where $\phi(z, t)$ is defined as the longitudinal wave potential and satisfies the relation

$$
\frac{\partial^{2} \phi}{\partial t^{2}}=c_{a}^{2} \frac{\partial^{2} \phi}{\partial z^{2}}
$$

where $c_{a}$ is the acoustic wave speed in the air. A general solution for the wave potential with arbitrary amplitude $A$, angular frequency $\omega$, and wave number $k_{a}$ is given as

$$
\phi(z, t)=A e^{i\left(k_{a} z-\omega t\right)}, \quad \text { with } k_{a}=\frac{\omega}{c_{a}} \quad \text { and } c_{a}=\frac{\lambda}{\rho} 1 / 2,
$$

where $\lambda$ is one of the Lame's constants and $\rho$ is the air density.

Similarly, the acoustic wave field in the two nanocomposite constituents can be described by the displacement function $u(z, t)$ determined by the Lame's constants and density of the two constituent layers as

$$
c_{i}=\left(\frac{\lambda_{i}+2 \mu_{i}}{\rho_{i}}\right)^{1 / 2}, \quad i=1,2 .
$$

The normal strain and the normal stress are assumed to be continuous at interfaces in the layered structures. As a result, the interfacial boundary conditions can be expressed as $[u]$ $=0$ and $\left[T_{33}\right]=0$, where $T_{33}$ is the normal tensile stress and can be written in terms of wave potential as

$$
T_{33}=(\lambda+2 \mu) \frac{\partial u}{\partial z}=(\lambda+2 \mu) \frac{\partial^{2} \phi}{\partial z^{2}} .
$$

Based on this, the boundary conditions at the interfaces can be written as

$$
\left[\frac{\partial \phi}{\partial z}\right]=0, \quad\left[(\lambda+2 \mu) \frac{\partial^{2} \phi}{\partial z^{2}}\right]=0 .
$$

In the layered structure, the wave in each layer is considered to be the summation of a forward propagation wave and a backward reflective wave. Based on this consideration, the one-dimensional wave potential can be expressed as

$$
\phi_{i}=A_{i} e^{i\left(k_{i} z_{i}-\omega t\right)}+B_{i} e^{i\left(-k_{i} z_{i}-\omega t\right)}, \quad V_{i}=B_{i} / A_{i},
$$

where $V_{i}$ is defined as the reflection coefficient.

When considering a composite to contain a total number of $L$ layers, following relationships can be established:

$$
\begin{aligned}
& \phi_{0}=A_{0} e^{i\left(k_{0} z-\omega t\right)}+B_{0} e^{i\left(-k_{0} z-\omega t\right)}, \\
& \phi_{1}=A_{1} e^{i\left(k_{1} z-\omega t\right)}+B_{1} e^{i\left(k_{1} z-\omega t\right)}, \\
& \phi_{2}=A_{2} e^{i\left(k_{2} z_{2}-\omega t\right)}+B_{2} e^{i\left(k_{2} z_{2}-\omega t\right)}, z_{2}=z-h_{1}, \\
& \phi_{L}=A_{L} e^{i\left(k_{L} z_{L}-\omega t\right)}+B_{L} e^{i\left(k_{L} z^{2}-\omega t\right)}, \\
& z_{L}=z-L \times h_{1} / 2-(L / 2-1) \times h_{2},
\end{aligned}
$$

where $k_{i}$ is the wave number in the $i$ th layer, and $h_{1}$ and $h_{2}$ are the thickness of the two constituent layers. For the transmitted wave back into the air, there is no reflection and the 
TABLE I. Density and mechanical property of PP, CNF-PP, and air. The density of CNF-PP is an approximation.

\begin{tabular}{lcccc}
\hline \hline & $\lambda(\mathrm{Pa})$ & $\mu(\mathrm{Pa})$ & Density $\left(\mathrm{kg} / \mathrm{m}^{3}\right)$ & Poisson' ratio \\
\hline PP & $6.3 \times 10^{8}-4.8 \times 10^{7} i$ & $2.9 \times 10^{8}-2.2 \times 10^{7} i$ & $1.2 \times 10^{3}$ & 0.34 \\
CNF-PP & $1.7 \times 10^{9}-1.1 \times 10^{8} i$ & $8.2 \times 10^{8}-5.3 \times 10^{8} i$ & $1.75 \times 10^{3}$ & 0.34 \\
Air & $1.01 \times 10^{5}$ & 0 & 1.2 & $\cdots$ \\
\hline \hline
\end{tabular}

wave potential only contains the forward propagation wave as

$$
\phi_{L+1}=A_{L+1} e^{i\left(k_{L+1} z_{L+1} \omega t\right)}, \quad z_{L+1}=z-L \times h_{1} / 2-L \times h_{2} / 2,
$$

where $k_{l+1}$ is the wave number in the air and a wave transmission coefficient $W$ of the whole structure can be defined using wave amplitude of the exiting and the incident wave as

$$
A_{L+1}=W \times A_{0} .
$$

The wave amplitude and stress amplitude at the $n$th interface can be expressed in terms of the coefficients of the potentials as

$$
\begin{aligned}
& u^{(n)}=i k_{n}\left(A_{n}-B_{n}\right), \\
& T_{33}^{(n)}=\left(\lambda_{n}+2 \mu_{n}\right)\left(-k_{n}^{2}\right)\left(A_{n}+B_{n}\right),
\end{aligned}
$$

where $n$ denotes the $n$th interface between $(n-1)$ th layer and $n$th layer. Based on above equations, the wave amplitudes and the stress amplitudes obey the relations

$$
\begin{aligned}
\left(\begin{array}{c}
u^{(n+1)} \\
T_{33}^{n+1}
\end{array}\right)= & \left(\begin{array}{cc}
\cos k_{n} h_{n} & \frac{\sin k_{n} h_{n}}{\left(\lambda_{n}+2 \mu_{n}\right) k_{n}} \\
-\left(\lambda_{n}+2 \mu_{n}\right) k_{n} \sin k_{n} h_{n} & \cos k_{n} h_{n}
\end{array}\right) \\
& \times\left(\begin{array}{c}
u^{(n)} \\
T_{33}^{(n)}
\end{array}\right) .
\end{aligned}
$$

Based on the 2-2 matrix of $M^{(n)}$ as defined above, relation between two layers can be established as

$$
\left(\begin{array}{l}
u^{(n)} \\
T_{33}^{(u)}
\end{array}\right)=M^{(n-1)} M^{(n-2)} \ldots M^{(1)}\left(\begin{array}{l}
u^{(1)} \\
T_{33}^{(1)}
\end{array}\right)=N^{(n)}\left(\begin{array}{l}
u^{(1)} \\
T_{33}^{(1)}
\end{array}\right) .
$$

Wave amplitude can now be written as

$$
\begin{aligned}
A_{n}= & \frac{1}{2}\left(Q_{11}^{(n)}+Q_{12}^{(n)}+Q_{21}^{(n)}+Q_{22}^{(n)}\right) A_{0}+\frac{1}{2}\left(-Q_{11}^{(n)}+Q_{12}^{(n)}\right. \\
& \left.+Q_{21}^{(n)}+Q_{22}^{(n)}\right) B_{0}, \\
B_{n}= & -\frac{1}{2}\left(Q_{11}^{(n)}+Q_{12}^{(n)}+Q_{21}^{(n)}+Q_{22}^{(n)}\right) A_{0}+\frac{1}{2}\left(-Q_{11}^{(n)}+Q_{12}^{(n)}\right. \\
& \left.+Q_{21}^{(n)}+Q_{22}^{(n)}\right) B_{0},
\end{aligned}
$$

where

$$
\begin{aligned}
& Q_{11}^{(n)}=\frac{k_{0}}{k_{n}} N_{11}^{(n)}, \\
& Q_{12}^{(n)}=i \frac{k_{0}^{2}}{k_{n}}\left(\lambda_{0}+2 \mu_{0}\right) N_{12}^{(n)},
\end{aligned}
$$

$$
\begin{aligned}
& Q_{21}^{(n)}=-i \frac{k_{0}}{k_{n}^{2}\left(\lambda_{n}+2 \mu_{n}\right)} N_{21}^{(n)}, \\
& Q_{22}^{(n)}=\frac{k_{0}^{2}\left(\lambda_{0}+2 \mu_{0}\right)}{k_{n}^{2}\left(\lambda_{n}+2 \mu_{n}\right)} N_{22}^{(n)},
\end{aligned}
$$

Solving Eqs. (17) and (18) gives the expressions for the coefficients of wave potentials in an arbitrary layer. $n=L+1$ define the last interface between the composite and air. As discussed previously, for the transmission wave $A_{L+1}=W$ $\times A_{0}$ and $B_{L+1}=0$. The numerical results for the transmission coefficients have been described in the next section.

\section{NUMERICAL ANALYSIS RESULTS AND DISCUSSION}

Physical properties of polypropylene (PP) and CNFreinforced PP (CNF-PP) were used as an example for acoustic wave propagation analysis. Since there is no CNF sheet reinforced PP data available, data for PP with uniformly distributed CNF as listed in Table I were adopted for calculation. ${ }^{27}$ Due to the different material properties, discontinuity in acoustic impedance is introduced at the layer interface. The wave speed was calculated to be 1.01 $\times 10^{3} \mathrm{~m} / \mathrm{s}$ in PP and $1.39 \times 10^{3} \mathrm{~m} / \mathrm{s}$ in CNF-PP. A reflection coefficient, defined as the ratio between the difference of the two acoustic impedances and the sum of the acoustic impedances, is calculated to be 0.33 . The increase in the number of layers can affect the transmission coefficient. In the calculation, the total composite sample thickness is fixed to be $1 \mathrm{~cm}$. In each repetitive bilayer, thickness of the PP layer is set to be four times that of the CNF-PP layer. For example, when $n=1$, there is one layer of PP with a thickness of $0.002 \mathrm{~m}$ and one layer of CNF-PP with a thickness of $0.008 \mathrm{~m}$ in the composite.

Figure 3 shows the calculated wave transmission coefficients as a function of the number of layer repeats for increasing acoustic wave frequencies. In the calculation, it is found that the sequence of two layers has no effects on the results. As shown in Fig. 3, the wave transmission coefficient has increasing number of oscillations for higher frequencies before converges to a stable value. This indicates that the layer structure can actually modulate the wave propagation and the resulted transmission coefficient can be controlled to certain extent with fixed total polymer and CNF contents.

The transmission coefficient modulation effect is closely related to the acoustic wavelength in the layers and the respective layer thickness. Table II lists the wavelengths in the PP and CNF-PP layers at different wave frequencies. With an incident wave angular velocity of $\omega=10^{5} \mathrm{rad} / \mathrm{s}$, both $\lambda_{\mathrm{PP}}$ 


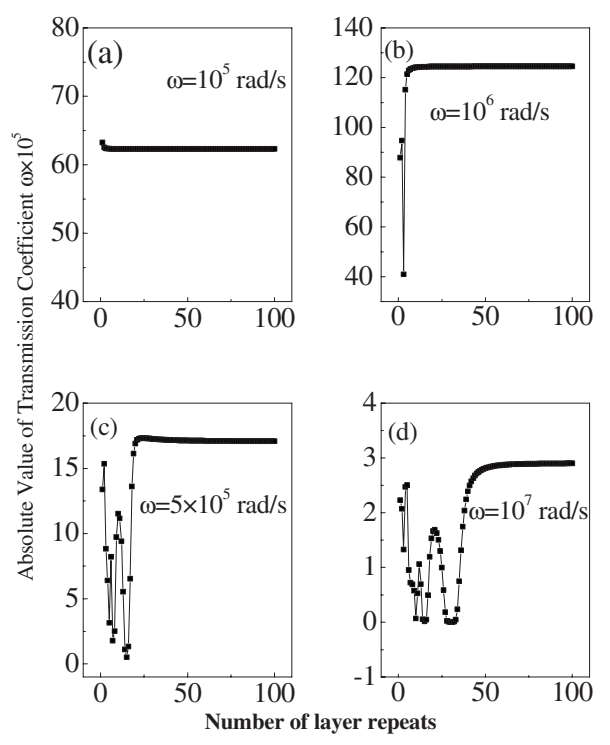

FIG. 3. Calculated acoustic wave transmission coefficient vs the number of layer repeats in the composite for different wave frequencies.

and $\lambda_{\text {CNF-PP }}$ are much larger than the total thickness of the structure. With increasing number of repeats, the PP/CNF-PP bilayer thickness decreases, and no modulation of transmission coefficient can be identified [Fig. 3(a)]. On the other hand, when the incident wave angular velocity increases to $\omega=10^{7} \mathrm{rad} / \mathrm{s}$, the wavelengths in the materials become comparable or smaller than the layer thickness when there is a small number of repeats. In this region, the effects of layered structure become significant and lead to transmission coefficient modulation [Figs. 3(b)-3(d)]. With increase number of layers and a fixed total sample thickness, the structural modulation effects will disappear and the transmission coefficient stabilizes. In other words, the analysis indicates that in the CNF paper sheet reinforced polymer composites, the bilayer thickness becomes the important length scale, and modulation on the wave propagation can happen at much lower frequencies than in the composites with uniformly distributed nanofillers.

According to the traditional wave propagation theory, ${ }^{8}$ when the acoustic wavelength is much longer than the bilayer thickness, the effects of the finely separated interfaces can be neglected and the sample can be treated as a homogenous effective media. The physical properties of the effective media are calculated from the volume fraction of the two constituent layers. Figure 4 shows the transmission coefficient calculated from the effective media theory. Clear agreement can be found when compared to the stable transmission coefficients obtained from layered structure analyses.

In the numerical analysis, mechanical properties of the materials reported by other researchers were used. In reality, viscoelastic properties of materials should exhibit frequency

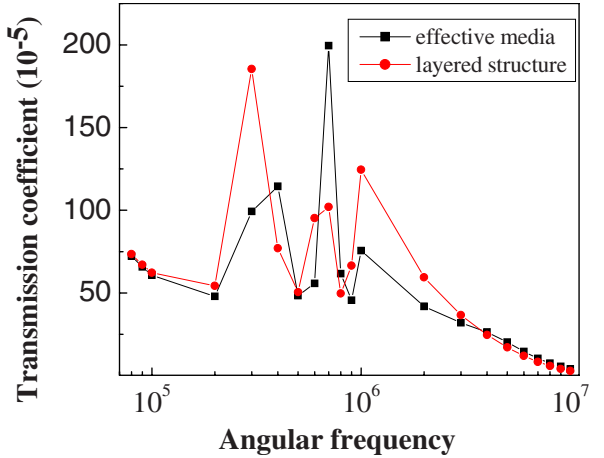

FIG. 4. (Color online) Comparison of the calculated long wavelength transmission coefficients based on layered structure and the effective media theory.

dependences which could quantitatively affect the analysis results. Normally, dynamic mechanic analyzer or dynamic mechanic thermal analyzer manufactured are used to characterize the viscoelastic properties of polymers. However, these measurements can only provide low frequency (less than several hundreds hertz) data. High frequency vibration measurements are needed to characterize the dynamic properties of the materials.

\section{CONCLUSIONS}

A normally incident plane harmonic acoustic wave propagation through a layered nanocomposite structure is studied. The calculated acoustic wave transmission coefficient oscillates when layer repeat thickness is comparable to wavelength. This indicates the possibility of control noise reduction by optimizing the layered structure. The study also shows that when the thickness of the layer repeat in composite is much smaller than the wavelength, the interface impedance mismatch will not have significant effects on the wave propagation and acoustic damping can be analyzed by considering the layered structure as a homogeneous effective media. There are reports ${ }^{28,29}$ on the wave propagation in layered media consisting of alternative layers of steel and plastics. It is also observed that when the wavelength is much larger than the thickness of the layers, the medium behaves as a homogeneous material. Also, strong transmission attenuation has been observed at high frequencies. We are currently synthesizing layered structures with controlled polymer and CNF composite layers for measurements.

The current model analyzes the normal incident wave across a one-dimensional layered structure where only longitudinal wave propagates in the material. A more general case should be the propagation of an obliquely incident wave. Under such a condition, in the air, still only exists the longitudinal wave; but both longitudinal and transverse

TABLE II. Acoustic wavelength in the pure PP and nanofiber-reinforced PP layers with various $\omega$

\begin{tabular}{lllllll}
\hline \hline$\omega(\mathrm{rad} / \mathrm{s})$ & $10^{5}$ & $5 \times 10^{5}$ & $10^{6}$ & $5 \times 10^{6}$ & $10^{7}$ & $5 \times 10^{7}$ \\
\hline$\lambda_{\text {PP }}(\mathrm{m})$ & 0.06 & 0.013 & 0.006 & 0.0013 & 0.0006 & 0.0001 \\
$\lambda_{\mathrm{CNF}-\mathrm{PP}}(\mathrm{m})$ & 0.09 & 0.018 & 0.009 & 0.0018 & 0.0009 & 0.0002 \\
\hline \hline
\end{tabular}


waves will be exited in the layered composite. Further study of the angular dependent acoustic wave transmission is needed.

\section{ACKNOWLEDGMENTS}

Financial support from TcSUH and National Science Foundation (CMMI-0620897) is gratefully acknowledged.

${ }^{1}$ J. W. Gillespie Jr., J. B. De Vault, D. D. Edie, V. Gabara, T. J. Haulik, High-Performance Structural Fibers for Advanced Polymer Matrix Composites (National Academy of Sciences, Washington, DC, 2005).

${ }^{2}$ N. A. Koratkar, B. Q. Wei, and P. M. Ajayan, Compos. Sci. Technol. 63, 1525 (2003).

${ }^{3}$ N. A. Koratkar, B. Q. Wei, and P. M. Ajayan, Adv. Mater. (Weinheim, Ger.) 14, 997 (2002).

${ }^{4}$ N. A. Koratkar, J. Suhr, A. Joshi, R. S. Kane, L. S. Schadler, P. M. Ajayan, and S. Bartolucci, Appl. Phys. Lett. 87, 063102 (2005).

${ }^{5}$ X. Zhou, E. Shin, K. W. Wang, and C. E. Bakis, Compos. Sci. Technol. 64, 2425 (2004).

${ }^{6}$ S. A. Suarez, R. F. Gibson, C. T. Sun, and S. K. Chaturvedi, Exp. Mech. 26, 175 (1986)

D. Qian, E. C. Dickey, R. Andrews, and T. Rantell, Appl. Phys. Lett. 76, 2868 (2000).

${ }^{8}$ P. M. Ajayan, L. S. Schadler, S. C. Giannaris, and A. Rubio, Adv. Mater. (Weinheim, Ger.) 12, 750 (2000).

${ }^{9}$ K. Lozano and E. V. Barrera, J. Appl. Polym. Sci. 79, 125 (2001); 80, 1162 (2001).

${ }^{10}$ R. Haggenmueller, H. H. Gommans, A. G. Rinzler, J. E. Fischer, and K. I. Winey, Chem. Phys. Lett. 330, 219 (2000).

${ }^{11}$ C. Park, Z. Ounaies, K. A. Watson, R. E. Crookes, J. Smith, S. E. Lowther,
J. W. Connell, E. J. Siochi, J. S. Harrison, and T. L. S. Clair, Chem. Phys. Lett. 364, 303 (2002).

${ }^{12}$ H. Kishi, M. Kuwata, S. Matsuda, T. Asami, and A. Murakami, Compos. Sci. Technol. 64, 2517 (2004).

${ }^{13}$ C. F. Ying and R. Truell, J. Appl. Phys. 27, 1086 (1956).

${ }^{14} \mathrm{P}$. M. Morse and K. U. Ingard, Theoretical Acoustics (Princeton University Press, Princeton, NJ, 1986).

${ }^{15}$ L. L. Foldy, Phys. Rev. 67, 107 (1945).

${ }^{16}$ M. Lax, Phys. Rev. 85, 621 (1952).

${ }^{17}$ P. C. Waterman and R. Truell, J. Math. Phys. 2, 512 (1961).

${ }^{18}$ J. T. Verbis, S. E. Kattis, S. V. Tsinopoulos, and D. Polyzos, Comput. Mech. 27, 244 (2001)

${ }^{19}$ Z. Wang, Z. Y. Liang, B. Wang, C. Zhang, and L. Kramer, Composites, Part A 35, 1225 (2004).

${ }^{20}$ M. Zhang and R. H. Baughman, Science 309, 1215 (2005).

${ }^{21}$ H. Xing, L. Sun, G. B. Song, J. Gou, and Y. W. Hao, Nanotechnology 19, 025704 (2008)

${ }^{22}$ J. Gou, S. O’Braint, H. C. Gu, and G. B. Song, J. Nanomater. 2006, 32803 (2006).

${ }^{23}$ M. S. Kushwaha, P. Halevi, L. Dobrzynski, and B. Djafari-Rouhani, Phys. Rev. Lett. 71, 2022 (1993)

${ }^{24}$ T. Gorishnyy, C. K. Ullal, M. Maldovan, G. Fytas, and E. L. Thomas, Phys. Rev. Lett. 94, 115501 (2005).

${ }^{25}$ L. M. Brekhovskikh, Waves in Layered Media, 2nd ed. (Academic, New York, 1980).

${ }^{26}$ A. Bedford and D. S. Drumheller, Introduction to Elastic Wave Propagation (Wiley, New York, 1994).

${ }^{27}$ I. C. Finegan, G. G. Tibbetts, and R. F. Gibson, Compos. Sci. Technol. 63, 1629 (2003).

${ }^{28}$ D. Marion and P. Coudin, 62nd Annual International Meeting of Society Exploration Geophysicists, 1992 (unpublished), p. 1341 (Expanded Abstract).

${ }^{29}$ J. M. Hovem, Geophysics 60, 1217 (1995). 